Chapter 24

\title{
The Role of The Angiosome Model in Treatment of Critical Limb Ischemia
}

\author{
Kim Houlind and Johnny Christensen \\ Additional information is available at the end of the chapter \\ http://dx.doi.org/10.5772/54418
}

\section{Introduction}

Critical limb ischemia (CLI) is the major cause of amputation in the developed world but revascularization offers an opportunity for limb salvage. Revascularization can be performed either by bypass surgery or by endovascular techniques. Peripheral bypass surgery can be performed using artificial grafts, but vein grafts offer better limb salvage and graft patency [1].

When performing revascularization of the lower limb, common clinical practice and recent guidelines include grafting of the" best vessel" which crosses the level of the ankle in order to restore pulsatile flow to the foot [1]. This may lead to either direct perfusion of the ischemic area or - very often - indirect perfusion relying on collaterals surrounding the diseased zone. This strategy is different from the one used e.g. in coronary artery bypass surgery, where the aim is "complete revascularization" i.e. performing bypasses to every diseased vascular territory [2].

The arterial connections between different parts of the foot may quite often not be sufficient to ensure healing and to prevent amputation. For instance, approximately $15 \%$ of heel ulcers do not heal despite an open bypass graft to the dorsal pedal artery [3]

An alternative strategy, called the angiosome model, is based on the pioneering work of Taylor and coworkers [4], who, in the eighties, performed detailed dissections with injection of dye in the vessels. They demonstrated the fact that the body consists of "angiosomes" i.e. three-dimensional blocks of tissue perfused and drained by specific arterial and venous bundles. In a later report from the same group, the angiosomes of the leg and foot were described in detail [5] 
Perfusion and drainage can occur between angiosomes by means of connecting "choke" vessels, but this perfusion is less effective than direct supply from the specific feed artery of the angiosome. It is worth noting that the choke vessels are diseased in patients with diabetes and atherosclerosis. This angiosome has had profound impact on the developement of strategies for plastic and reconstructive surgery. However, only little attention has been paid to the angiosome model in treatment of critical limb ischemia. According to the angiosome model, the specific feed artery - rather than the "best vessel" - should be favoured for revascularization. The foot and ankle area consist of six angiosomes.

During the last few years, some studies have compared the results of "best vessel" versus "angiosome" directed revascularization. The studies include comparisons of both arterial bypass and percutaneous revascularization based on the two principles

This chapter aims at describing the role of the angiosome model in critical limb ischemia, and to review the current literature.

\section{Anatomy}

Blood supply to the foot is derived from the three tibial vessels, the Anterior tibial artery, the Posterior tibial artery, and the Peroneal artery. These three arteries give rise to six endarteries, each supplying an angiosome (Figure 1).

1. The anterior tibial artery supplies the anterior ankle and continues as the dorsalis pedis artery, which supplies the dorsum of the foot. It gives off the lateral tarsal artery and branches into the first dorsal interosseal artery and the arcuate artery supplying the 2-4 interosseal arteries. It has been pointed out that the dorsalis pedis artery is extremely attenuated or absent in $12 \%$ of cases [6].

The posterior tibial artery divides into three main branches:

2. The calcaneal branch, which arborizes into multiple braches, that supply the medial and plantar portion of the heel,

3. the medial plantar artery, supplying the medial, plantar part of the foot. Its boundaries encompass the instep, and, depending on anatomic variability, can include the hallux.

4. the lateral plantar artery which supplies the lateral midfoot as well as the entire plantar forefoot through the 4 plantar metatarsal arteries that emanate from the deep plantar arch. Normally, this angiosome also includes the plantar aspect of the hallux, depending on anatomic variability.

The peroneal artery bifurcates into

5. the anterior perforating brach, supplying the lateral anterior upper ankle and

6. a calcaneal branch, supplying the lateral and plantar heel. Together with the calcaneal brach of the posterior tibila artery this artery ensures a double blood supply to the plantar aspect of the heel. 

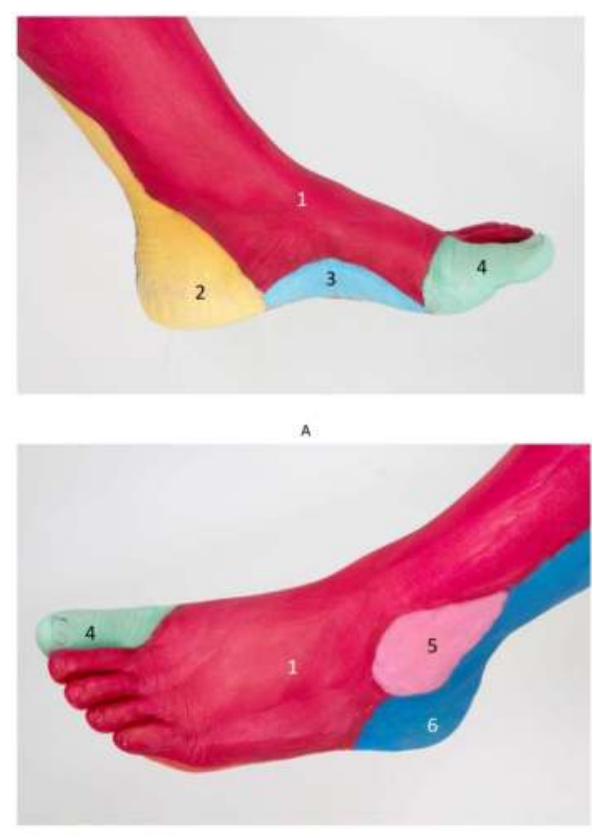

8

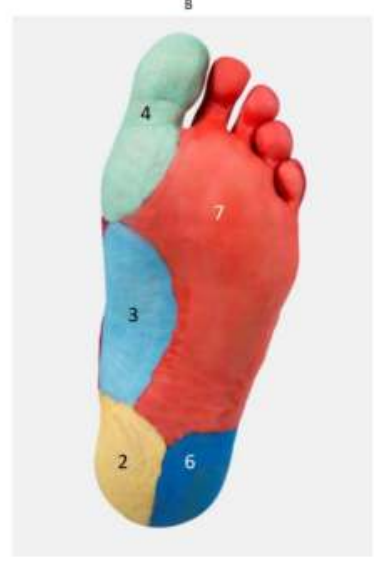

c

1. Dorsalis pedis angiosome

2. Medial calcaneal artery angiosome

3. Medial plantar artery angiosome

4. The hallux, which may be supplied by the feeding arteries of angiosomes 1, 2, or 6

5. Anterior perforating branch angiosome

6. Lateral calcaneal branch angiosome

7. Lateral plantar artery angiosome

Figure 1. Angiosomes shown on the surface of the foot. A. Medial view, B. Dorso-lateral view, C. Plantar view. 


\section{Interconnections}

A number of interconnections exist between the angiosomes. When present, these interconnections exist a priori and - in contrast to the choke vessels described below - do not need a period of ischemia to open. However, as peripheral arterial disease progresses, these connections may be blocked.

The arterial-arterial connections include:

Anterior tibial to peroneal:

The lateral malleolar artery joins with the anterior perforating branch of the peroneal artery just above the ankle joint (Figure 2A).

\section{Anterior tibial to posterior tibial:}

The lateral plantar artery forms the deep plantar arch crossing the proximal 2,3, and 4th metatarsals and finally anastomoses directly with the dorsalis pedis artery in the first interspace (Figures $2 \mathrm{~A}$ and $2 \mathrm{~B}$ ). The superficial and deep medial plantar arteries join at the cruciate anastomosis. Depending on what arteries predominate at or around the cruciate anastomosis, the hallux may be primarily nourished by the lateral plantar artery, medial plantar artery, the first dorsal metatarsal artery or simultaneously by either two or three of these arteries [7].

The medial plantar artery also interconnects with the anterior tibial tree as cutaneous branches connect proximally with medial branches of the dorsalis pedis artery and distally with branches of the first dorsal metatarsal artery.

Peroneal and posterior tibial connections:

Between one and three communicating branches between the peroneal artery and the posterior tibial artery proximal to the ankle joint deep to the Achilles tendon.

On the other hand, no direct arterial-arterial connection exists between the medial and lateral calcaneal arteries, which both supply the plantar aspect of the heel.

\section{Choke vessels}

Where no "true" arterial-arterial connections are present between neighbouring angiosomes, a network of reduced caliber "choke vessels" form a link. These vessels are normally inadequate to perfuse the area of a distant angiosome but may be provoked to dilate.

This is the theoretical base of the "delay phenomenon" which has been applied in plastic surgery. While the choke vessels between angiosomes in a skin or muscle flap may be sufficient to perfuse an adjacent vascular territory, necrosis will ususally appear in the choke vessel zone defining the next vascular territory. When designing a skin or muscle flap larger than two angiosomes, a two stage procedure might be performed. In the first stage, the perforators of the neighbouring angiosomes are ligated, causing the choke vessels between neighbouring angiosomes to dilate over a period of 4-10 days. After this delay period, a larger flap can be safely elevated [8]. There is good clinical and experimental evidence that this 
principle works for the transfer of skin grafts from essentially normal donor sites. These results may, however, not be extrapolated to other situations e.g. in the ischemic foot where distal, aggressive macroangiopathy is associated with microcirculatory changes like thrombosis, neuropathy, local sepsis, arterio-venous shunting and hypercoagulability [9].

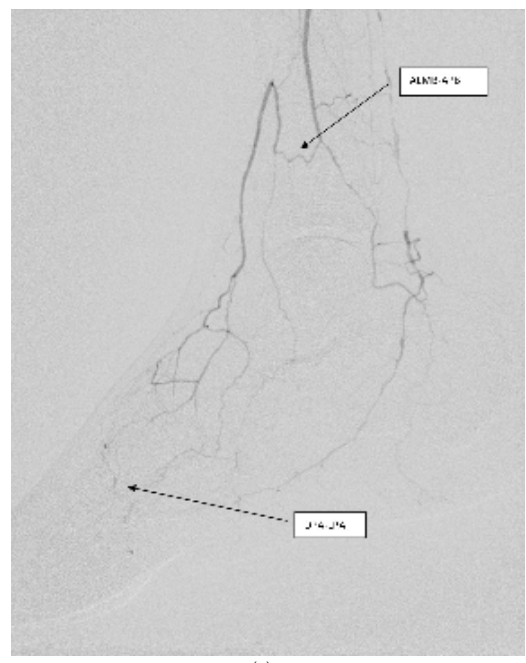

(a)

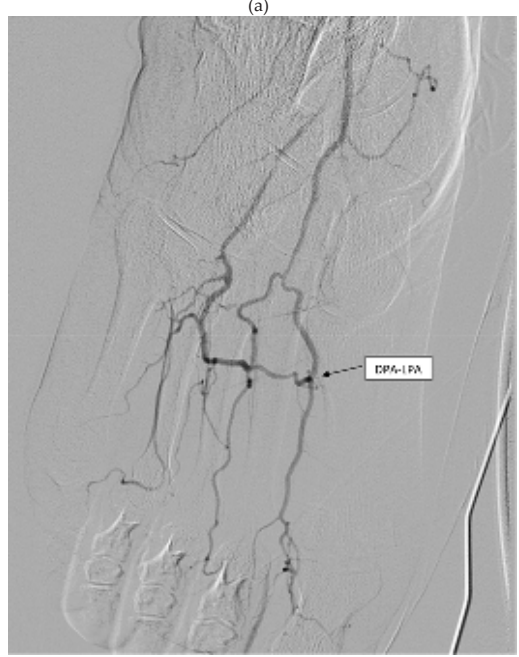

Figure 2. A. Lateral oblique projection of the anterior pedal vessels of a patient with peripheral occlusive arterial disease and patent arterial-arterial connections. ALMB-APB: Connection between the anterior lateral malleolar branch of the anterior tibial artery and the anterior perforating branch of the peroneal artery. DPA-LPA: Perforating branch connecting the dorsal pedal artery with the lateral plantar artery. B. Antero-posterior projection of the perforating branch connecting the dorsal pedal artery with the lateral plantar artery (DPA-LPA). 


\section{Imaging and assessment}

\subsection{Angiography}

A fundamental prerequisite of providing angiosome-directed revascularization is profound knowledge of the anatomy of the pedal vasculature as well as adequate imaging technique including intraprocedural angiography of both tibial and pedal arteries. Manzi and coworkers have recently reported their experience from more than 2500 antegrade interventional procedures in patients with critical limb ischemia and diabetes [10]. For imaging of the pedal arteries they stress that prolonged filming is often necessary to record delayed enhancement of of pedal vessels from retrograde or collateral circulation and that both standard anteroposterior and lateral oblique projections should be obtained. They have established the following two criteria for correct positioning of the image intensifier: 1) The base of the fifth metatarsal bone must be seen to project outward from the base of the foot in the lateral oblique view and 2) the first proximal metatarsal interspace must be clearly visualized in the anteroposterior view. These two views tend to give a good overview of the pedal arteries and collaterals.

\subsection{Doppler ultrasound}

Attinger and coworkers have described in detail how to map the arterial-arterial connections using a Doppler device [7].

As an example, the Doppler signal is located from the posterior tibial artery over the tarsal tunnel. If the signal persists when occluding (by digital compression) the artery distally, there is antegrade flow along the posterior tibila artery. If the signal disappears, the flow is retrograde from the anterior tibial artery via the dorsalis pedis and lateral plantar arteries. Similarly, Doppler signal can be obtained from the anterior perforating branch of the peroneal artery in the lateral soft area between the tibia and fibula just above the ankle joint. When the anterior tibial artery is occluded at the takeoff of the lateral malleolar branch, the Doppler signal will persist if there is antegrade flow along the anterior perforating branch of the peroneal artery. If the Doppler signal disappears, filling of the anterior perforating branch must be retrograde from the anterior tibial artery through the lateral malleolar branch. The authors describe how the competence of these connections can have profound significance for the healing potential of an amputation wound.

\subsection{Thermography}

Nagase and coworkers [11] reported the results of plantar thermography of skin temperature in 129 non-ulcer diabetic patients and 32 normal volunteers. From the pattern of four different plantar angiosomes originally described by Attinger [7], they defined twenty different patterns of temperature distribution. The most common pattern in normal subjects was a "bilateral butterfly pattern" in which the medial arch showed the highest temperature $(46.9 \%)$ or an even distribution of temperature across the entire planta of the feet $(20.3 \%)$. 
Recordings of the diabetic feet showed a lower proportion of feet with a "bilateral butterfly pattern" (13.9\%), higher proportions of even distribution of temperature (39.1\%) and a generally more diverse distribution of patterns in the rest. Although interesting, the study did not provide comparisons with angiographic findings that could confirm a correlation between the distribution of skin temperature and the distribution of lesions of feed arteries to the relevant angiosomes.

\section{Results from direct versus indirect revascularization}

A number of studies have been performed comparing the results of direct revascularization to the relevant angiosome with those of indirect revascularization either through collaterals or choke vessels.

In 2009, Neville and coworkers published a retrospective analysis of 43 patients undergoing bypass surgery for tissue loss due to ischemia [12]. Twenty-two were directly revascularized to the relevant agniosome while 21 were indirectly revascularized. Healing occurred in $91 \%$ of the directly revascularized patients and only $62 \%$ of the indirectly revascularized patients $(\mathrm{p}=0.03$ ]. Major patient characteristics such as diabetes, tobacco use, and renal failure were evenly distributed between the directly revascularized and indirectly revascularized groups, but wound characteristics and infection were not reported.

On the other hand, Azuma and coworkers [13] reviewed the results of 249 consecutive distal bypasses for critical limb ischemia. 218 limbs were included in the initial analysis which proved significantly lower wound healing rate in the indirect revascularization group than in the direct revascularization group. This was especially the case in a subgroup of patients with end stage renal failure. This finding was, however, compromised by significant baseline differences between the groups especially characterized by a higher proportion of patients with heel ulcers and gangraene in the indirect revascularization group. After applying propensity scored analysis including only 48 pairs of limbs, the healing rate between the two groups did not reach statistical significance $(p=0.185)$. The authors concluded that the angiosome concept was not relevant for open surgical treatment of critical limb ischemia in patients without end stage renal failure. This conclusion may be questioned in view of the limited statistical strength of the propensity scored analysis.

Iida and coworkers reviewed the results of endovascular treatment of 203 limbs in 177 consecutive patients with critical limb ischemia, Rutherford 5 or 6 [14]. During up to 4 years follow up, they found significantly higher limb salvage rate in patients with the directly revascularized than indirectly revascularized wounds. Interestingly, the total number of tibial vessels with run off did not influence the limb salvage rate in neither group, indicating that it is not important how much blood can be provided to the foot but rather whether i $t$ reaches the ischemic area. In a later review by the same group [15], including 369 limbs from 329 consecutive patients, including only patients with isolated below-the-knee lesions, patients who had received direct revascularization experienced significantly higher levels of amputation-free survival and freedom from major adverse limb events than patients in 
whom only indirect revascularization was possible. In this review the finding was confirmed after propensity matching of groups. In multivariate analysis, elevated levels of c-reactive protein were found to be independent predictors of major amputation in the indirect revascularization group but not in the direct revascularization group. This may imply that indirect revascularization may be inadequate for the healing of infected wounds.

Alexandriescu and collegues have published several reports describing their experience with targeted primary angioplasty of diabetic foot lesions [16-17]. In a series of 124 limbs (98 patients), they were able to achieve direct revascularization in $82 \%$ [16]. Limb salvage was $91 \%$ at 12 months and $84 \%$ at three years follow-up. More recently, they published a historical comparison between their results before and after 2005 when they introduced the angiosome concept in their practice. Despite similar graft patency and technical success, they experienced a significantly better wound healing rate and limb preservation in the group of patients treated according to the angiosome concept [18]. This result is interesting although it is probably biased by the general learning curve of the group.

In a paper published together with Alexandriescu, the vascular surgery department at the University Hospital in Helsinki, Finland recently reported their results from the last three years [19]. In a population including approximately the same number of direct and indirect endovascular revascularizations, they found $74 \%$ of the wounds to have healed within one year in the directly revascularized group compared to $46 \%$ in the indirectly revascularizd group $(\mathrm{p}=0.002)$. The number of patients was, however, not reported.

Two studies, one surgical by Deguchi [20] and one endovascular by Blanes Ortí [21] failed to show any difference in wound healing time or limb salvage between directly or indirectly revascularized patients. Due to small numbers, the statistical strengh of these comparisons is, however, limited.

\subsection{The influence of collaterals}

The prognostic significance of indirect revascularization via collaterals was studied by Varela in a mixed cohort of venous bypass and endovascular treated patients with ischemic wounds [22]. Defining collaterals visible on perioperative angiograms, either between distal calcaneal peroneal branches and anterior or posterior tibial artery $(n=16)$ or patent pedal arch connecting dorsal and plantar blood supply $(n=2)$, they found a similar wound healing rate for indirect revascularization of the wound area through collaterals as for direct revascularization to the angiosome specific feed artery (92\% versus $88 \%$ wound healing at 12 months follow-up). When including indirect revascularizations without visible collaterals, only $73 \%$ of the wounds had healed after 12 months ( $p=0.008)$.

\subsection{The significance of venosomes}

Anatomically, the venous drainage follows the arterial perfusion of the angiosomes [23] and Alexandriescu used the term venosome, when reporting the results of surgical deep calf vein arterialization. In a series of 26 limbs in 25 diabetic patients with very advanced below the knee occlusive disease, a PTFE bypass was made between an arterial inflow and a deep 
calf vein followed by selective embolization of collaterals, directing arterial blood to the relevant venosome. Using this strategy, a 73\% three year limb salvage rate was achieved [24].

\section{Discussion}

The concept of angiosome-directed revascularization is, theoretically, attractive and in accordance with pathophysiological knowledge. It is also in line with experience from coronary bypass surgery, where reperfusion through collaterals does not provide a similar freedom from cardiac events as that provided by complete direct revascularization of all the diseased vascular territoria [2].

It is well established that healing of an ishemic pedal wound is more effectively achieved when pulsatile arterial blood flow is established across the ankle and it seems logical to expect that this effect is larger when the pulsatile flow is provided all the way to the site of the injury.

As suggested by the above mentioned papers, the effect of direct revascularization may especially be relevant in the settings of end stage renal failure, infected wounds, endovascular rather than surgical repair, and in cases where collaterals are absent.

The angiosome concept represents a novel approach to improving the therapy of critical limb ischemia. It may potentially provide the rationale not only for the choice of target artery. It may also influence the indications for endovascular or open repair according to which target artery is accessible by which method.

Although the evidence in favour of an angiosome directed treatment is mounting fast, it is, however, still circumstantial. All of the studies comparing the results of direct and indirect revascularization are retrospective and, thus, biased by heterogeneity in patient selection. More often than not, the angiosome specific artery will also be the most diseased artery and the ability to recanalize this vessel will most probably select the least atherosclerotic patients to the "direct revascularization" group. It is also likely that the advocates of an angiosomedirected revascularization strategy would attempt direct revascularization first and only perform indirect revascularization if this attempt was unsuccessful. Regardless of any retrospective matching of the groups this would lead to patients with extensive distal atherosclerosis to be placed in the indirect revascularization groups, thus biasing the comparisons in favour of the angiosome specific approach. The differences in healing rate and limb salvage between groups may, therefore, merely reflect preoperative differences in the extent of occlusive disease. It is possible that this is what is reflected in the lack of statistically significant differences after propensity scoring in the study by Azuma [13].

As highlighted in the study by Varela, the presence or absence of collaterals merit further investigation [22]. For this purpose, the Doppler method described by Attinger [7] seems to be a good and non-invasive technique.

As evidence stands at the moment, there is some, although limited, evidence that when there is a choice of target artery for revascularization, preference should be given to the ar- 
tery directly feeding the wound's angiosome. Specific analysis, based on prospectively collected data of homogeneous cohorts of patients are needed. Unbiased evidence will only be achievable by performing a prospective, randomized controlled trial with a blinded endpoint assessment.

\section{Author details}

Kim Houlind ${ }^{1}$ and Johnny Christensen ${ }^{2}$

1 Dept. of Vascular Surgery, Kolding Hospital, Denmark and Institute of Regional Health Services Research, University of Southern Denmark, Denmark

2 Dept. of Radiology, Kolding Hospital, Denmark

\section{References}

[1] Norgreen L, Hiatt WR, Dormandy JA, Nehler MR, Harris KA, Fowkes FGR on behalf of the TASC II Working Group. Inter-society Concensus for the management of peripheral arterial disease (TASC II). Eur J Endovasc Surg 2007;33(Suppl 1): S32-55.

[2] Vieira RD, Hueb W, Gersh BJ, Lima EG, Pereira AC, Rezende PC, Garzillo CL, Hueb AC, Favarato D, Soares PR, Ramires JA, Filho RK. The effect of complete revascularization on 10-year Survival of Patients with Stable Multivessel Coronary artery Disease: MASS II Trial. Circulation 2012;126 (11 Suppl 1): S158-63

[3] Berceli SA, Chan AK, Pomposelli FB jr. Gibbons GW, Campbell DR, Akbari CM, Brophy DT, LoGerfo FW. Efficacy of dorsal pedal artery bypass in limb salvage for ischemic heel ulcers. J Vasc Surg 1999;30(3):499-508

[4] Taylor GI, Palmer JH. The vascular territories (angiosomes) of the body: Experimental study and clinical implication. Br J Plast Surg 1987;40:113-

[5] Taylor GI, Pan WR. Angiosomes of the leg: anatomic study and clinical implications. Plast Reconstr Surg 1998;102:599-616

[6] Clemens MW, Attinger CE. Angiosomes and wound care in the diabetic foot. Foot Ankle Clin N Am 2010;15:439-64

[7] Attinger CE, Evans KK, Bulan E, Blume P, Cooper P. Angiosomes of the Foot and Ankle and Clinical Implications for Limb Salvage: Reconstruction, Incisions, and Revascularization. Plast. Reconstr. Surg 2006; 117 (Suppl) 261S-293S

[8] Taylor GI, Corlett RJ, Caddy CM, Zeit RG. An anatomic review of the delay phenomenon: II. Clinical applications. Plast Reconstr Surg:89 (3):408-16 
[9] Jörneskog G. Why critical limb ischemia criteria are not applicable to diabetic foot and what the consequences are. Scand J Surg 2012:101;114-18

[10] Manzi M, Cester G, Palena LM, Alek RT, Candeo A, Ferraresi R. Vascular Imaging of the Foot: The first step toward endovascular recanalization. Radiographics 2011;31(6):1623-36

[11] Nagase T, Sanada H, Takehara K, Oe M, Iizaka S, Ohashi Y, Oba M, Kadowaki T, Nakagami G. Variations of planatar thermographic patterns in normal controls and non-ulcer diabetic patients: Novel classification using angiosome concept. Journal of Plastic, reconstructive \& aesthetic Surgery 2011; 64: 860-66

[12] Neville RF, attinger CE, Bulan EJ, Ducic I, Thomassen M, Sidaway AN. Revascularization of a Specific Angiosome for Limb salvage: Does the Target artery Matter? Ann Vasc Surg 2009;23:367-373

[13] Azuma N, Uchida H, kokubo T, Koya A, Akasaka N, sasajima T. factors influencing wound healing og critical ischaemic foot after bypass surgery: Is the angiosome important in selecting bypass traget artery. European journal of vascular and endovascular surgery 2012;43:322-328

[14] Iida O, Nanto S, Uematso M, Ikeoka K, Okamoto S, Dohi T, Fujita M, Terasi H, Nagata S. Importance of the angiosome concept for endovascular therapy in patients with critical limb ischemia. Catheterization and cardiovascular Interventions 2010; 75: 830-836

[15] Iida O, Soga Y, Hirano K, Kawasaki D, Suzuki K, Miyashita Y, Terasi H, Uematsu M. Long term reults of direct and indirect endovascular revascularization base don the angiosome concept in patients with critical limb ischemia presenting with isolated below-the knee lesions. J Vasc surg 2012;55:363-70

[16] Alexandriescu V-A, Hubermont G, Philips Y, Guillaumie B, Ngongang C, Vandenbossche P, Azdad K, Ledent G, Horion J. Selective primary angioplasty following an angiosome model of reperfusion in the treatment of Wagner 1-4 diabetic foot lesions: Practice in a multidisciplinary diabetic limb service. J Endovasc Ther 2008;15:580-593

[17] Alexandriescu V, Hubermont G. The challenging topic of diabetic foot revascularization: does the angiosome-guided angioplasty may improve outcome. Journal of Cardiovascular Surgery 2012;53:3-12.

[18] Alexandriescu V, Vincent G, Azdad K, Hubermont G, Ledent G, Ngongang C, Filimon A-M. A reliable approach to diabetic neuroischemic foot wounds: Below-theknee angiosome-oriented angioplasty. J Endovasc Ther 2011;18:376-387

[19] Alexandriescu V, Söderström M, Venermo M. Angiosome theory: Fact or fiction? Scandinavian Journal of surgery 2012;101:125-31

[20] Deguchi J, Kitaoka T, Yamamoto K, Matsumoto H, Sato. Impact of Angiosome on Treatment of Diabetic Ischemic Foot with Paramalleolar Bypass. J Jpn Coll Angiol 2010;50:687-691 
[21] Blanes Orti P, Vázquez R, Minguell P, García V, Manuel-Rimbau Munoz E, Lozarno Vilardell P. Percutaneous revascularization of specific angiosome in critical limb ischaemia. Angiologia 2011;63:11-17

[22] Varela C, Acin F, de Haro J, Bleda S, Esparza L, March JR. The role of foot collateral vessels on ulcer healing and limb salvage after successful endovascular and surgical distal procedures according to the angiosome model. Vasc Endovasc Surg 2010;44:654-660

[23] Taylor G.I, Caddy CM, Waterson PA, Crock JG. The venous territories (venosomes) of the human body. Experimental study and clinical implications. Plast Reconstr Surg 1990;86:185-

[24] Alexandriescu V, Ngongang C, Vincent G, Ledent G, Hubermont G. Deep calf veins arterialization for inferior limb preservation in diabetic patients with extended ischemic wounds, unfit for direct arterial reconstruction; preliminary results according to an angiosome model of perfusion. Cardiovascular revascularization Medicine 2011;12:10-19 